\title{
Computational modeling of volumetric soft tissue growth: application to the cardiac left ventricle
}

\author{
Wilco Kroon • Tammo Delhaas • Theo Arts • \\ Peter Bovendeerd
}

Received: 5 September 2007 / Accepted: 26 July 2008 / Published online: 30 August 2008

(C) The Author(s) 2008. This article is published with open access at Springerlink.com

\begin{abstract}
As an initial step to investigate stimulus-response relations in growth and remodeling (G\&R) of cardiac tissue, this study aims to develop a method to simulate 3Dinhomogeneous volumetric growth. Growth is regarded as a deformation that is decomposed into a plastic component which describes unconstrained growth and an elastic component to satisfy continuity of the tissue after growth. In current growth models, a single reference configuration is used that remains fixed throughout the entire growth process. However, considering continuous turnover to occur together with growth, such a fixed reference is unlikely to exist in reality. Therefore, we investigated the effect of tissue turnover on growth by incrementally updating the reference configuration. With both a fixed reference and an updated reference, strain-induced cardiac growth in magnitude of $30 \%$ could be simulated. However, with an updated reference, the amplitude of the stimulus for growth decreased over time, whereas with a fixed reference this amplitude increased. We conclude that, when modeling volumetric growth, the choice
\end{abstract}

W. Kroon $(\varangle) \cdot$ T. Delhaas

Department of Physiology, Cardiovascular Research Institute

Maastricht, Maastricht University, PO Box 616,

6200 MD Maastricht, The Netherlands

e-mail: jw.kroon@fys.unimaas.nl

T. Arts

Department of Biophysics, Cardiovascular Research Institute Maastricht, Maastricht University, PO Box 616,

6200 MD Maastricht, The Netherlands

T. Delhaas

Department of Pediatrics, Cardiovascular Research Institute Maastricht, University Hospital Maastricht, PO Box 5800, 6202 AZ Maastricht, The Netherlands

P. Bovendeerd

Department of Biomedical Engineering, Eindhoven University

of Technology, PO Box 513, 5600 MB Eindhoven, The Netherlands of the reference configuration is of great importance for the computed growth.

Keywords Cardiac mechanics - Remodeling - Adaptation . Finite element method

\section{Introduction}

Over the years several finite element (FE) models of cardiac mechanics have been developed to study pump function in relation to the three-dimensional (3D) geometrical, passive, active and anisotropic properties of the myocardium (Bovendeerd et al. 1992; Costa et al. 2001; Geerts-Ossevoort 2003; Kerckhoffs et al. 2003; Nash and Hunter 2000; Usyk et al. 2002; Stevens et al. 2003). Tissue properties, however, are not constant over time as the tissue exhibits growth and remodeling (G\&R) in response to (changes in) mechanical loading (Donker et al. 2005; Holmes 2004; MacKenna et al. 2000; Sadoshima and Izumo 1997; Simpson et al. 1999). The clinically most evident examples are left ventricular (LV) hypo- or hypertrophy in response to hemodynamic under- or overloading of the LV, respectively. Furthermore, local changes in mechanical loading, as induced by asynchronous contraction, have been shown to result in asymmetric wall thickening (van Oosterhout et al. 1998). The details on the type of stimulus and the local response, however, still remain unclear (Omens 1998; Humphrey 2001). Extension of models of cardiac mechanics with features of G\&R may provide additional insight in adaptation by allowing quantitative testing of hypotheses regarding the stimulus-response relation. Subsequently, thus extended models may be used to estimate long-term outcome of clinical interventions causing chronic changes in load, such as cardiac re-synchronization therapy. 
Recently, 3D FE models have been developed that enable computation of volumetric growth in patient specific geometries (Alastrue et al. 2007; Kuhl et al. 2007; Ramasubramanian et al. 2006; Rodriguez et al. 2007). In these models, volumetric growth is regarded as a deformation that potentially changes the externally unloaded shape, volume and state of stress of the tissue (Rodriguez et al. 1994; Skalak et al. 1982; Taber 1998). Internal stresses are constitutively related to growth deformation via an initial stress-free configuration of the tissue that remains fixed throughout the entire growth process. As such, growth remains dependent on the initial tissue configuration.

In an alternative approach, the tissue has been considered as a mixture of constituents, each of which exhibits continuous turnover (Humphrey and Rajagopal 2002). An implication of this turnover is that a certain initial configuration becomes irrelevant once all initial tissue constituents have been renewed. From this argument, constitutive laws used in growth models to relate internal stresses to growth deformation should not be related to a fixed reference configuration, but rather to a configuration that evolves together with growth.
The aim of the present study is twofold. The first aim is to include volumetric growth in an existing 3D model of cardiac mechanics. The second aim is to investigate the effect of using a fixed or an evolving stress-free reference on progression of volumetric growth. Both approaches are implemented in a 3D FE framework.

\section{Method}

\subsection{Growth during a single growth increment}

Assuming density of soft tissue to be constant, growth is modeled by a change of volume. As shown in Fig. 1, a growth increment from time $t=t_{0}$ to time $t=t_{0}+\Delta t$ is simulated by deforming the stress-free tissue configuration $\mathcal{B}_{0}$ to the externally unloaded, not necessarily internally stress-free, grown configuration $\mathcal{B}_{g}$ in three steps.

Step 1: Computation of the growth stimulus In the first step, the externally loaded tissue configuration $\mathcal{B}_{l}$ is determined. Deformation is quantified by the load deformation gradient

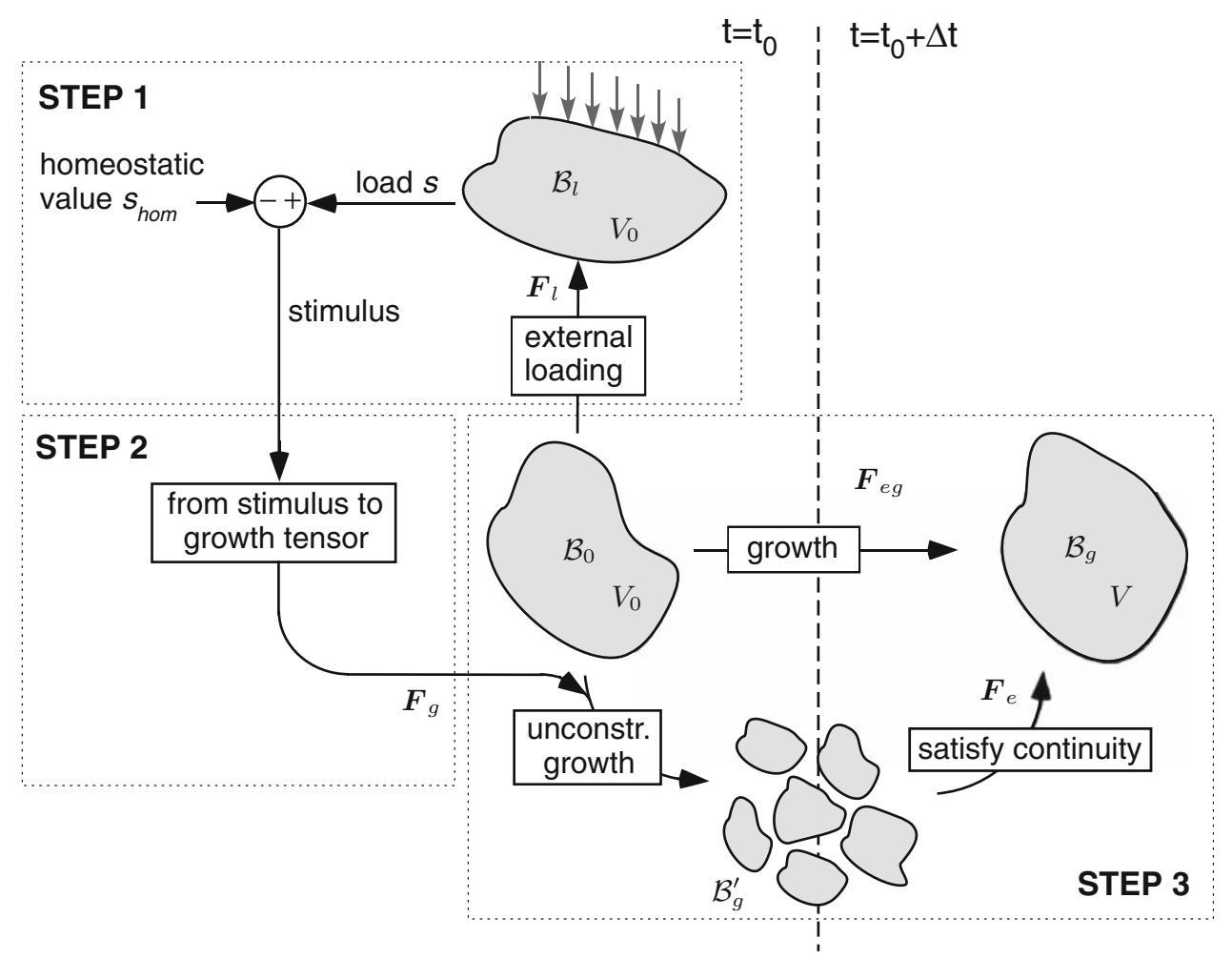

Fig. 1 Schematic description of a growth increment. Simulation of a growth increment from time $t=t_{0}$ to time $t=t_{0}+\Delta t$ involves three steps. In step 1 , externally unloaded configuration $\mathcal{B}_{0}$ with volume $V_{0}$ is externally loaded, resulting in configuration $\mathcal{B}_{l}$. In this configuration a load measure $s$ is extracted and the growth stimulus is determined from deviation of $s$ from a homeostatic value $s_{\text {hom }}$. In step 2 the stimulus is translated into a growth tensor $\boldsymbol{F}_{g}$ that describes local unconstrained growth of the unloaded tissue to the intermediate configuration $\mathcal{B}_{g}^{\prime}$. To satisfy continuity of the grown tissue, an additional deformation $\boldsymbol{F}_{e}$ is required that generates internal stresses. In step $3, \boldsymbol{F}_{g}$ is used to compute the combined growth deformation $\boldsymbol{F}_{e g}=\boldsymbol{F}_{e} \cdot \boldsymbol{F}_{g}$ from equilibrium of linear momentum, yielding grown configuration $\mathcal{B}_{g}$ with volume $V$ 
tensor $\boldsymbol{F}_{l}$, which is related to tissue displacements $\vec{u}_{l}$ through:

$$
\boldsymbol{F}_{l}=\left(\vec{\nabla}_{0} \vec{u}_{l}\right)^{T}+\boldsymbol{I}
$$

with $\vec{\nabla}_{0}$ the spatial gradient operator with respect to the stress-free configuration $\mathcal{B}_{0}$ and $\boldsymbol{I}$ the identity tensor. Under external loading conditions, Cauchy stress $\sigma$ in the tissue is constitutively related to deformation $\boldsymbol{F}_{l}$ by a strain energy density function $W_{l}$ :

$\boldsymbol{\sigma}\left(\boldsymbol{F}_{l}\right)=\frac{1}{\operatorname{det}\left(\boldsymbol{F}_{l}\right)} \boldsymbol{F}_{l} \cdot \frac{\partial W_{l}\left(\boldsymbol{E}_{l}\right)}{\partial \boldsymbol{E}_{l}} \cdot \boldsymbol{F}_{l}^{T}$

where $\boldsymbol{E}_{l}=\frac{1}{2}\left(\boldsymbol{F}_{l}^{T} \cdot \boldsymbol{F}_{l}-\boldsymbol{I}\right)$ is the Green-Lagrangian strain due to external loading. Displacements are computed from balance of linear momentum, which under the assumption of negligible body and inertial forces reads:

$\vec{\nabla}_{l} \cdot \boldsymbol{\sigma}\left(\boldsymbol{F}_{l}\right)=\overrightarrow{0}$ on $\mathcal{B}_{l}$

where $\vec{\nabla}_{l}$ denotes the spatial gradient operator with respect to $\mathcal{B}_{l}$. The problem definition is completed with natural and essential boundary conditions along deformed surface parts $\partial \mathcal{B}_{l, n}$ and $\partial \mathcal{B}_{l, e}$, respectively:

$\boldsymbol{\sigma}\left(\boldsymbol{F}_{l}\right) \cdot \vec{n}=\vec{t}_{p} \quad$ on $\partial \mathcal{B}_{l, n}$

$$
\vec{u}=\vec{u}_{p} \quad \text { on } \partial \mathcal{B}_{l, e}
$$

with $\vec{t}_{p}$ a prescribed traction and $\vec{u}_{p}$ a prescribed displacement.

Next, a scalar load measure $s$ is extracted from $\mathcal{B}_{l}$. Similar to Taber (1998) we assume growth to be stimulated if the load $s$ differs from its homeostatic (i.e. target) value $s_{\text {hom, }}$, i.e.:

stimulus $=$ function $\left(s-s_{\text {hom }}\right)$

Step 2: Translation of the stimulus to a growth tensor In a second step, the stimulus is translated into an incremental growth deformation tensor $\boldsymbol{F}_{g}$ that describes unconstrained tissue growth.

Local volume changes during growth are mathematically specified by the determinant of $\boldsymbol{F}_{g}$ :

$\operatorname{det}\left(\boldsymbol{F}_{g}\right)=\frac{V}{V_{0}}$

with $V_{0}$ and $V$ the local tissue volumes before and after the growth increment. The isochoric part $\tilde{\boldsymbol{F}}_{g}=\operatorname{det}\left(\boldsymbol{F}_{g}\right)^{-\frac{1}{3}} \boldsymbol{F}_{g}$ specifies the preferred direction in which the new tissue is deposited. In this study, without loss of generality of the kinematic description, new tissue constituents are assumed to be deposited in all directions equally (isotropic growth) and $\boldsymbol{F}_{g}$ takes the form:

$\boldsymbol{F}_{g}=\left(\frac{V}{V_{0}}\right)^{\frac{1}{3}} \boldsymbol{I}$

The idea that a supra-homeostatic load will lead to a net increase of volume, whereas a sub-homeostatic load will lead to a decrease of volume, is phenomenologically described by:

$\dot{V}=\beta V\left(s-s_{\text {hom }}\right)$

with $\beta$ a rate constant. Linearization with respect to volume $V_{0}$ at the start of the growth increment yields:

$\frac{V}{V_{0}}=\beta\left(s-s_{\mathrm{hom}}\right) \Delta t+1$

Consequently, for the incremental growth deformation tensor it holds:

$\boldsymbol{F}_{g}=\left[\beta\left(s-s_{\text {hom }}\right) \Delta t+1\right]^{\frac{1}{3}} \boldsymbol{I}$

Step 3: Computation of growth displacements In a third step, the growth tensor $\boldsymbol{F}_{g}$ is translated into growth displacements $\vec{u}_{e g}$. Deformation according to $\boldsymbol{F}_{g}$ leads to the fictitious intermediate configuration $\mathcal{B}_{g}^{\prime}$ in which local tissue parts have expanded (Fig. 1). Generally, an additional deformation is required to ensure that locally grown tissue parts form an intact tissue configuration $\mathcal{B}_{g}$. This deformation is quantified by $\boldsymbol{F}_{e}$. Consequently, a tensor $\boldsymbol{F}_{e g}$ is defined that transforms the unloaded configuration $\mathcal{B}_{0}$ into the grown configuration $\mathcal{B}_{g}$ :

$\boldsymbol{F}_{e g}=\boldsymbol{F}_{e} \cdot \boldsymbol{F}_{g}$

Change in tissue volume is related to $\boldsymbol{F}_{g}$ only, i.e. $\boldsymbol{F}_{e}$ is isochoric.

Growth displacements are related to $\boldsymbol{F}_{e g}$ according to:

$\boldsymbol{F}_{e g}=\left(\vec{\nabla}_{0} \vec{u}_{e g}\right)^{T}+\boldsymbol{I}$

Generally, part $\boldsymbol{F}_{g}$ is assumed to be plastic and stresses are only generated by the elastic part $\boldsymbol{F}_{e}$ (Alastrue et al. 2007; Kuhl et al. 2007; Ramasubramanian et al. 2006; Rodriguez et al. 1994, 2007; Skalak et al. 1982; Taber 1998). In our model, the growth-induced Cauchy stress $\boldsymbol{\sigma}_{g}\left(\boldsymbol{F}_{e}\right)$ is related to a strain energy density function $W_{g}$ through:

$\boldsymbol{\sigma}_{g}\left(\boldsymbol{F}_{e}\right)=\frac{1}{\operatorname{det}\left(\boldsymbol{F}_{e}\right)} \boldsymbol{F}_{e} \cdot \frac{\partial W_{g}\left(\boldsymbol{E}_{e}\right)}{\partial \boldsymbol{E}_{e}} \cdot \boldsymbol{F}_{e}^{T}$

with $\boldsymbol{E}_{e}=\frac{1}{2}\left(\boldsymbol{F}_{e}^{T} \cdot \boldsymbol{F}_{e}-\boldsymbol{I}\right)$. Growth displacements are computed from balance of linear momentum, which is now given by:

$\vec{\nabla}_{g} \cdot \boldsymbol{\sigma}_{g}\left(\boldsymbol{F}_{e}\right)=\overrightarrow{0} \quad$ on $\mathcal{B}_{g}$

where $\vec{\nabla}_{g}$ denotes the spatial gradient operator with respect to the intact grown configuration $\mathcal{B}_{g}$. Growth is assumed to alter the externally unloaded (traction-free) tissue configuration. To model this, boundary conditions are specified according to:

$$
\begin{aligned}
\boldsymbol{\sigma}_{g}\left(\boldsymbol{F}_{e}\right) \cdot \vec{n} & =\overrightarrow{0} & & \text { on } \partial \mathcal{B}_{g, n} \\
\vec{u} & =\vec{u}_{p} & & \text { on } \partial \mathcal{B}_{g, e}
\end{aligned}
$$


Fig. 2 Configurations and deformations involved during successive load-induced growth increments. a The stress-free reference tissue configuration $\mathcal{B}_{0}$ remains fixed and internal stresses accumulate. b The stress-free reference configuration $\mathcal{B}_{0}$ is updated after each growth increment. Consequently, stresses will be present in the grown tissue, but they will not accumulate

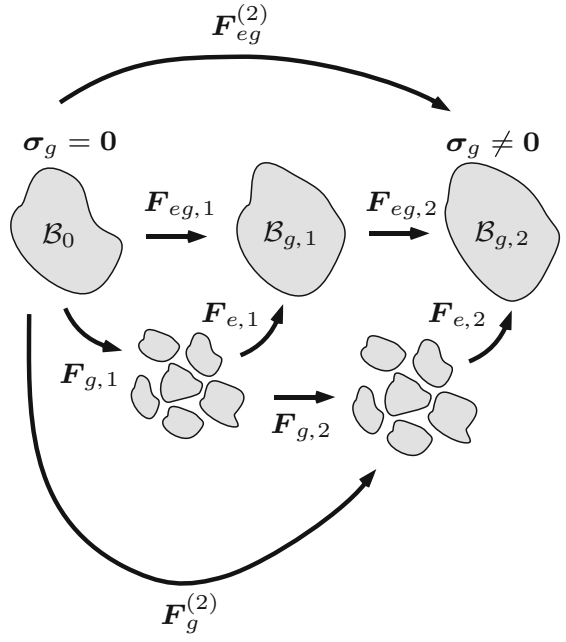

(a) fixed reference

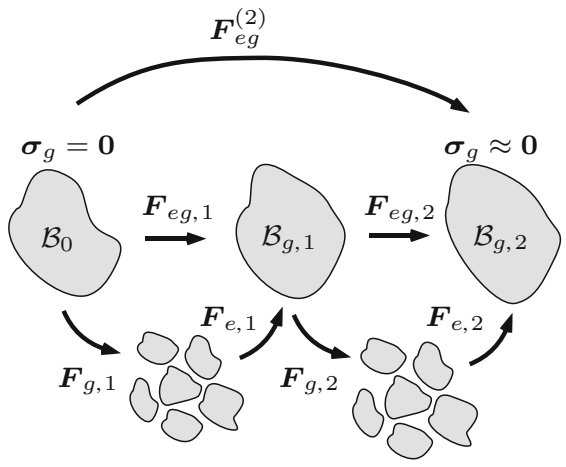

(b) updated reference

\subsection{Cumulative growth}

Generally, after one growth increment, the initial unloaded configuration $\mathcal{B}_{0}$ is deformed by $\boldsymbol{F}_{\text {eg }, 1}$ to form the new externally unloaded tissue configuration $\mathcal{B}_{g, 1}$ (Fig. 2), where the subscript is added to indicate the increment number. Next, a new growth increment is started by loading configuration $\mathcal{B}_{g, 1}$, resulting in externally loaded configuration $\mathcal{B}_{l, 1}$ where a stimulus is computed and translated into a new growth deformation $\boldsymbol{F}_{g, 2}$. Subsequently, $\boldsymbol{F}_{g, 2}$ is used to determine the new configuration $\mathcal{B}_{g, 2}$, which is related to $\mathcal{B}_{g, 1}$ through $\boldsymbol{F}_{e g, 2}$. As can be derived from Fig. 2, after $n$ growth increments $\mathcal{B}_{0}$ is cumulatively deformed into $\mathcal{B}_{g, n}$ according to $\boldsymbol{F}_{e g}^{(n)}$ which is given by:

$$
\begin{aligned}
\boldsymbol{F}_{e g}^{(n)} & =\boldsymbol{F}_{e g, n} \cdot \boldsymbol{F}_{e g, n-1} \cdots \boldsymbol{F}_{e g, 1} \\
& =\prod_{i=1}^{n} \boldsymbol{F}_{e g, i}
\end{aligned}
$$

To asses the effect of using a fixed or an updated stressfree configuration, we distinguish between two approaches to compute the cumulative growth tensor (Fig. 2a, b).

Fixed reference configuration Figure $2 \mathrm{a}$ schematically shows the commonly used approach in which the stressfree reference configuration $\mathcal{B}_{0}$ remains fixed throughout the growth simulation (Alastrue et al. 2007; Kuhl et al. 2007; Ramasubramanian et al. 2006; Rodriguez et al. 2007). In this approach, internal stresses after increment $n$ are related to $\boldsymbol{F}_{e, n}$ as given by:

$\boldsymbol{F}_{e, n}=\boldsymbol{F}_{e g}^{(n)} \cdot\left(\boldsymbol{F}_{g}^{(n)}\right)^{-1}$ with $\boldsymbol{F}_{g}^{(n)}$ the cumulative unconstrained tissue growth that is given by:

$\boldsymbol{F}_{g}^{(n)}=\prod_{i=1}^{n} \boldsymbol{F}_{g, i}$

In this approach, the cumulative grown deformation $\boldsymbol{F}_{e g}^{(n)}$ is directly determined from the balance of linear momentum in Eq. (14) by using $\boldsymbol{F}_{g}^{(n)}$ as input with stresses related to $\boldsymbol{F}_{e, n}$. Thus, internal stress in the grown configuration remains dependent on the initial configuration $\mathcal{B}_{0}$ through $\boldsymbol{F}_{e g}^{(n)}$.

Updated reference configuration In this case, growthinduced internal stresses are assumed to relax due to tissue turnover. To describe this behavior the stress-free reference configuration is updated after each growth increment using the computed incremental tissue displacements (Fig. 2b). Consequently, internal stress in the grown configuration is only dependent on the last grown configuration $\mathcal{B}_{g, n-1}$ through $\boldsymbol{F}_{e g, n}$. Now, for the elastic deformation $\boldsymbol{F}_{e, n}$ it holds:

$\boldsymbol{F}_{e, n}=\boldsymbol{F}_{e g, n} \cdot \boldsymbol{F}_{g, n}^{-1}$

In this approach, the incremental growth deformation $\boldsymbol{F}_{e g, n}$ is computed from the balance of linear momentum using the incremental unconstrained growth tensor $\boldsymbol{F}_{g, n}$ as input. The cumulative growth deformations $\boldsymbol{F}_{e g}^{(n)}$ and $\boldsymbol{F}_{g}^{(n)}$ are then determined by the incremental deformations according to (16) and (18), respectively.

\subsection{Application to the cardiac left ventricle}

Geometry We illustrate both approaches by simulating growth in the cardiac left ventricle (LV). The unloaded LV wall is represented by a truncated ellipsoid with a cavity and 
Fig. 3 Computational geometry of the left ventricle (LV). a The externally unloaded LV geometry is represented by a truncated ellipsoid. In this geometry, myofiber directions $\vec{e}_{f, 0}$ are quantified by angles $\alpha_{h, 0}$ with respect to the circumferential direction $\vec{e}_{\phi}$. endo endocardium, epi epicardium. b Finite element mesh containing tri-quadratic hexahedral elements consisting of 27 nodal points with 3 displacement degrees of freedom each
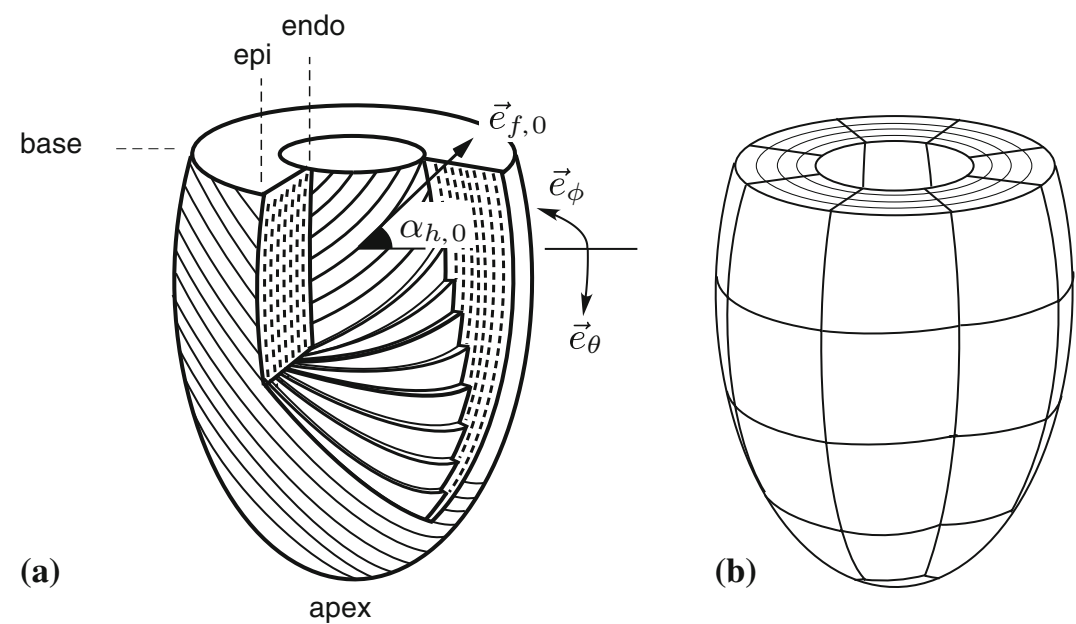

wall volume of 44 and $120 \mathrm{ml}$, respectively (Fig. 3a). Both are representative volumes for the dog LV (Bovendeerd et al. 1992).

Strain energy density function Under loading conditions, the passive cardiac tissue is strongly non-linear and anisotropic due to the fibrous structure. To model this behavior, we use the strain energy density function $W_{l}$ as proposed by Kerckhoffs et al. (2003):

$W_{l}=W_{m}+W_{f}+W_{v}$

with

$$
\begin{aligned}
& W_{m}=a_{0}\left[e^{a_{1} \operatorname{tr}\left(\boldsymbol{E}_{l}^{T} \cdot \boldsymbol{E}_{l}\right)}-1\right] \\
& W_{f}=a_{2}\left[e^{a_{3} E_{f f}^{2}}-1\right] \\
& W_{v}=a_{4}\left[\operatorname{det}^{2}\left(\boldsymbol{F}_{l}\right)-1\right]^{2}
\end{aligned}
$$

where objectivity of stress is ensured by choosing $W_{l}$ as a function of $\boldsymbol{E}_{l}$. Part $W_{m}$ describes the isotropic response due to extracellular matrix deformation, while $W_{f}$ describes additional stiffness in myofiber direction. The GreenLagrangian myofiber strain $E_{f f}$ is specified by $E_{f f}=\vec{e}_{f, 0}$. $\boldsymbol{E}_{l} \cdot \vec{e}_{f, 0}$. Near-incompressible behavior is enforced through $W_{v}$. Material parameter values $a_{0}=0.5 \mathrm{kPa}, a_{1}=3.0 \mathrm{kPa}$, $a_{2}=0.01 \mathrm{kPa}$ and $a_{3}=60.0 \mathrm{kPa}$ were obtained from Kerckhoffs et al. (2003). The bulk modulus $a_{4}$ was set to $5.0 \mathrm{kPa}$.

Myofiber orientation Myofiber orientation $\vec{e}_{f, 0}$ in the externally unloaded LV is quantified by helix angle $\alpha_{h, 0}$ with respect to a prolate ellipsoidal coordinate system (Fig. 3a):

$\vec{e}_{f, 0}=\cos \left(\alpha_{h, 0}\right) \vec{e}_{\phi}+\sin \left(\alpha_{h, 0}\right) \vec{e}_{\theta}$

where $\vec{e}_{\phi}$ and $\vec{e}_{\theta}$ denote the circumferential and the longitudinal direction in the unloaded geometry, respectively. Initially, the myofiber angle $\alpha_{h, 0}$ varies linearly in trans- mural direction from $+60^{\circ}$ at the endocardium to $-60^{\circ}$ at the epicardium. During growth, new tissue is assumed to locally have the same myofiber angle as that in the existing tissue. This implies that transmural heterogeneity in tissue growth affects the transmural course of the myofiber angle.

Stimulus and growth Growth is assumed to be triggered by a deviation of end-diastolic myofiber strain from a homeostatic value $s_{\text {hom }}=0.13$. This homeostatic value roughly resembles myofiber strain during the filling phase in the healthy LV. End-diastolic myofiber strain $s$ is given by:

$s=\sqrt{2 E_{f f}+1}-1$

The end-diastolic situation was simulated by imposing a surface traction $\vec{t}_{p}=-p \vec{n}$, with $\vec{n}$ the unit outer normal on the deformed surface and $p$ the cavity pressure of $1 \mathrm{kPa}$.

During growth, the product $\beta \Delta t$ in Eq. (10) was set to 1 . The strain energy density function was taken the same as during external loading, i.e. $W_{g}=W_{l}$.

Simulations and analysis Using both a fixed and an updated reference configuration to simulate growth, we assessed volume changes, changes in end-diastolic strains and changes in myofiber angles. The number of growth increments was chosen such that for both simulations, volume changed by about $30 \%$. Change in unloaded tissue volume was computed according to:

$$
\left(\operatorname{det}\left(\boldsymbol{F}_{e g}^{(n)}\right)-1\right) \times 100 \%
$$

Furthermore, to asses directionality of growth, we divided change in tissue volume into change of myofiber length computed according to:

$\left(\lambda_{e g, f}-1\right) \times 100 \%$ 
and change of area perpendicular to the myofiber (cross myofiber area) as computed from:

$$
\left(\frac{\operatorname{det}\left(\boldsymbol{F}_{e g}^{(n)}\right)}{\lambda_{e g, f}}-1\right) \times 100 \%
$$

where myofiber growth stretch ratio $\lambda_{e g, f}$ is given by:

$\lambda_{e g, f}=\sqrt{\vec{e}_{f, 0} \cdot C_{e g}^{(n)} \cdot \vec{e}_{f, 0}}$

with $C_{e g}^{(n)}=\boldsymbol{F}_{e g}^{(n)^{T}} \cdot \boldsymbol{F}_{e g}^{(n)}$ the right Cauchy growth strain.

\subsection{Numerical treatment}

At each time increment, tissue displacements due to external loading or growth are determined by solving the balance of linear momentum in Eqs. (3) and (14) with a Galerkintype of finite element method (Bathe 1982), as implemented in the commercial package SEPRAN (SEPRA, Leidschendam, The Netherlands). Prescribed displacements $\vec{u}_{p}$ are identical for the loading and growth step: basal movement is restricted in apex-to-base direction, while rotation of the endocardium with respect to the epicardium is allowed. Switching from computation of displacements during loading $\left(\vec{u}_{l}\right)$ to computation of displacements during growth $\left(\vec{u}_{e g}\right)$ involves a change in traction $\vec{t}_{p}$ by switching between cavity pressure $p$ from 1 to $0 \mathrm{kPa}$, respectively.

A modified Newton-Raphson method was employed to linearize balance of linear momentum with respect to the stress-free reference configuration (total Lagrangian formulation). Spatial discretization occurred with tri-quadratic hexahedral elements, while spatial integration was conducted with a Newton-Cotes scheme.

The mesh of the initial unloaded stress-free reference configuration is visualized in Fig. 3b. For the fixed reference approach, the finite element mesh remains the same for each growth increment. For the updated reference approach, the mesh is updated after a growth increment by displacing the

Fig. 4 Load-induced growth in the cardiac LV: global volume and shape changes. a Initial myofiber strain at end of diastole (cavity pressure $p=1 \mathrm{kPa}$ ).

b-c Cumulative volume changes in the grown geometry. The dashed contour indicates the initial geometry. The number of increments $n=t /(\beta \Delta t)$. Simulation end-times were selected upon volumetric growth being in magnitude of $30 \%$. Since the evolution of the stimulus differed between the two simulations, both have different end-times

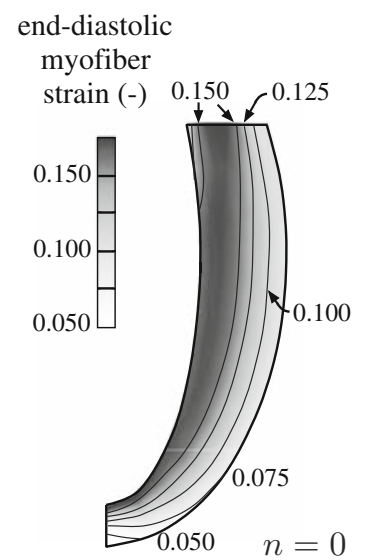

(a) before growth

nodal points by the incremental growth displacements $\vec{u}_{e g}$, while neglecting the internal stresses. This results in a new stress-free mesh.

\section{Results}

Figure 4a shows an inhomogeneous distribution of enddiastolic myofiber strain after the first loading step, as visualized in the initial unloaded geometry. End-diastolic myofiber strains are higher at the sub-endocardium than at the subepicardium.

Figure 4b, c shows the total amount of volume change, together with the change in geometry as obtained with a fixed reference and an updated reference, respectively. With both cases significant changes in volume could be obtained with a magnitude of $30 \%$. However, to achieve this change with an updated reference approach about twice as much increments were needed.

As growth progressed and the reference configurations in both approaches started to diverge, end-diastolic strain became different also. Figure 5 provides a more detailed view of the transmural distributions of tissue growth, end-diastolic strain, and helix angles before and after growth at three different longitudinal levels. The left panel of Fig. 5a shows that the pattern of local volume change is similar for the two approaches. When local volume change is divided into change in cross myofiber area and change in myofiber length, it becomes evident that volumetric growth predominantly occurs through a change in cross myofiber area (compare middle and right panel, Fig. 5a). With both approaches, cross myofiber area increases in the subendocardium and decreases in the subepicardium. Differences arise between both simulations, when regarding the change in myofiber length. With both approaches, subepicardial myofibers shorten. However, subendocardial myofibers shorten when using the updated

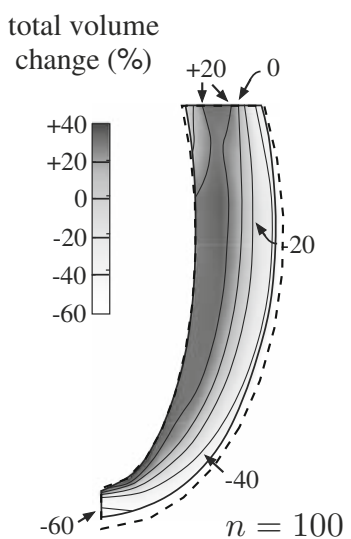

(b) after growth, fixed reference

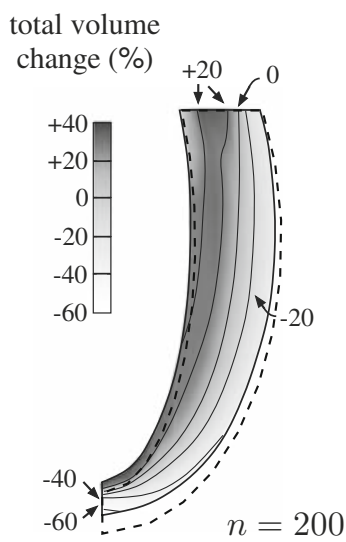

(c) after growth, updated reference 


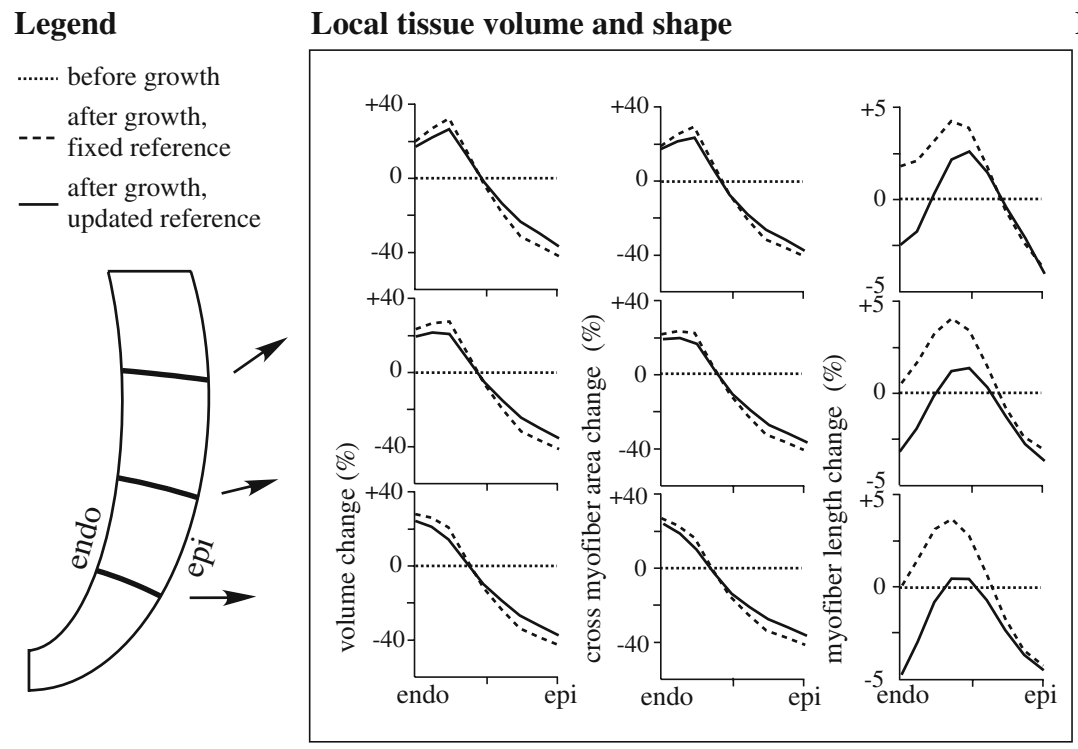

(a)
End-diastolic strain

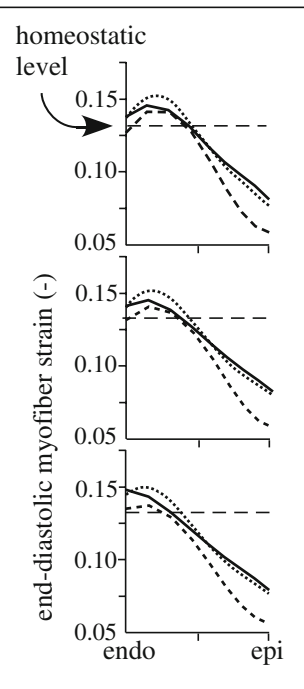

(b)
Local tissue structure

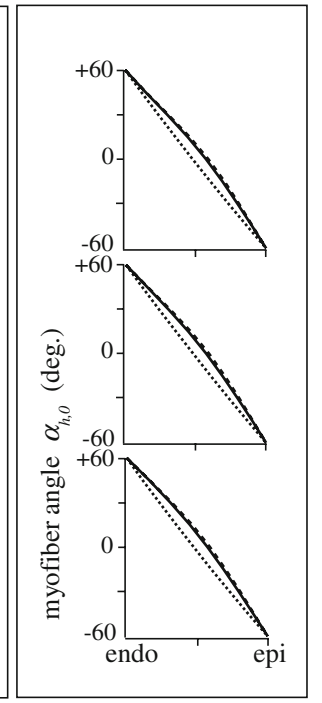

(c)

Fig. 5 Transmural distribution of growth at three different levels from endocardium (endo) to epicardium (epi). a Change in local tissue volume and shape. With both fixed and updated reference, similar volumetric growth is observed (left). The observed change in cross myofiber area is similar as well (middle), but differences are observed in subendocardial changes in unloaded myofiber length (right). Note the difference in scale between the middle and the right panel. Volume change predominantly occurs via a change in cross myofiber area. b Change in

reference approach, while they lengthen when using the fixed reference approach (right panel, Fig. 5a).

Growth using an updated reference caused end-diastolic myofiber strains over the complete transmural range to become closer to the homeostatic values (Fig. 5b). With a fixed reference, however, myofiber strains decreased over the whole transmural range, resulting in divergence from the homeostatic values in mid-wall and subepicardial regions.

As a consequence of the transmural inhomogeneity of growth, in both simulations the transmural distribution of the myofiber angles evolved from a linear to a more parabolic shape (Fig. 5c). Regions with positive growth exhibited a decreased transmural gradient of fiber angle $\alpha_{h, 0}$ in the externally unloaded configuration, whereas an increase in transmural gradient was observed in regions with negative growth.

\section{Discussion}

The aim of the present study was twofold. The first aim was to develop and test a method to simulate load-induced inhomogeneous three-dimensional volumetric growth of a complex geometry such as the cardiac LV. Generally, our approach to simulate a load-induced increment in volumetric growth involves three steps; (1) simulation of beat-to-beat tissue

end-diastolic myofiber strain. When updating the reference configuration, end-diastolic strain becomes closer to the homeostatic value over the whole transmural range. When fixing the reference, end-diastolic strains from mid-wall to epicardium diverge from the homeostatic value. c Due to inhomogeneous volumetric growth, the transmural distribution of myofiber angles $\alpha_{h, 0}$ in the externally unloaded tissue changes from a linear to a more parabolic one

mechanics to determine a load measure relevant for growth deviation of this measure from its homeostatic value yields the growth stimulus; (2) the stimulus is related to a desired amount of volume change, which is subsequently translated into a growth tensor $\boldsymbol{F}_{g}$ that describes unconstrained tissue growth; and (3) based on the growth tensor, a simulation of growth mechanics is used to deform the unloaded tissue configuration to an intact locally grown tissue. Commonly a fixed reference is used to compute growth during multiple increments. However, in light of continuous turnover occurring together with growth, such a fixed reference is unlikely to exist in reality. Therefore, as a second aim we investigated the effect of updating the stress-free reference after each growth step on the grown geometry.

To illustrate our model we simulated LV growth in response to end-diastolic myofiber strain. With both fixed and updated reference approach, similar growth behavior could be observed, as far as changes in tissue volume, cross myofiber area and myofiber orientation are concerned (Fig. 5). With both approaches, volume changes in magnitude of $30 \%$ could be obtained. However, end-diastolic strain developed differently between both approaches. While growth with an updated reference caused end-diastolic myofiber strain across the wall to develop towards to the homeostatic value, growth with a fixed reference resulted in mid-wall and subepicardial strains that were further apart from the homeostatic value. 
Apparently, our simulations have not progressed to a point where differences in end-diastolic strain between the two approaches clearly affect the observed volumetric and cross-sectional change. However, our results show that fixing or updating the reference configuration may eventually have a significant effect on the achieved growth.

It remains unclear which of the two approaches is more realistic. Fixing the reference configuration causes shape changes during growth to result in accumulation of internal stresses in the externally unloaded tissue. These residual stresses are usually assumed to cause the opening of the externally unloaded tissue upon incision. As such, comparison of model-predicted opening angles with experimentally determined ones has been used as a way to check the growth model (Taber and Humphrey 2001). Although the traditionally used growth model with a fixed reference provides for a theoretical link between growth and residual stress development, unfortunately however, to the best of knowledge, no direct experimental evidence exists that either supports or rejects a causal relation between growth and residual stress buildup. In contrast, when considering tissue turnover, stresses due to growth are more likely to be related to incremental growth rather than to accumulated growth, i.e. turnover causes constituents bearing the residual stresses to be replaced. Therefore, it may be more physiological to update the stress-free reference configuration after each growth increment, instead of fixing it. However, when updating the reference, build-up of residual stress during growth is limited to the last increment. To still account for the existence of residual stresses in real mature tissue we note that it has been hypothesized that, during turnover, new constituents may be added in the existing tissue under pre-stretch, thereby affecting the residual stress state of the total tissue (Humphrey and Rajagopal 2002; Watton et al. 2004). As such, residual stresses may originate from a combination of G\&R.

Experimental data remain unclear about the relation between the growth stimulus and the response (Humphrey 2001; Omens 1998). As a consequence, several assumptions had to be made in the growth model. Firstly, we used enddiastolic myofiber strain as growth trigger, since in vitro stretching of myocytes has been shown to induce a hypertrophic response (Sadoshima and Izumo 1997; Simpson et al. 1999). However, experimental data remain inconclusive and other candidates remain plausible. For instance, growth due to increased filling in vivo was also found to correlate to ejection (myofiber) strain and end-diastolic stress (Donker et al. 2005; Holmes 2004). To be more specific, Donker et al. (2005) found a correlation between normalization of ejection strain and end-diastolic stress on the one hand, and a decrease in growth rate on the other. As a approximation, we adopted a linear relation between the growth rate and the stimulus (Eq. 4).
Secondly, we assumed unconstrained growth to be isotropic, since no experimental data exists about the directionality of unconstrained tissue growth. Experimental data shows that, depending on the loading conditions, the intact cardiac LV can exhibit eccentric growth, in which mainly the length of the myofibers increases, or concentric growth, in which mainly the thickness of the myofibers increases (Gerdes and Capasso 1995). Interestingly, our simulations show that isotropic unconstrained growth yields an anisotropic, growth pattern in the intact heart, as volume changes predominantly occurred by a change in myofiber cross-sectional area (Fig. 5a). Should experimental data about the directionality of growth become available, it can readily be incorporated by changing the form of growth tensor $\boldsymbol{F}_{g}$ (Ramasubramanian et al. 2006, Rodriguez et al. 2007).

Thirdly, we assumed strain energy density functions during loading $\left(W_{l}\right)$ and growth $\left(W_{g}\right)$ to be the same. However, as stated by (Humphrey and Rajagopal 2002): "a constitutive relation does not define a material, it merely quantifies a behavior under certain circumstances". In other words, the short-term behavior of cardiac tissue on a beat-to-beat time scale may differ from the behavior on a time scale of growth, suggesting $W_{l} \neq W_{g}$.

Finally it is noted that, in our model, we implicitly included the effect of turnover by updating the reference. In the future, the growth model should be extended with more detailed description of turnover and remodeling, capable of including an explicit relation between (changes in) local tissue behavior (e.g. orientation, stiffness, pre-stretch, etc.) and (changes in) local volume. The theory of mixtures as proposed by Humphrey and Rajagopal (2002) provides an elegant approach to do this. We envisage that such an extension enables the model to capture a much wider range of physiological processes, thereby potentially generating additional insights into the relation between local mechanical stimuli and G\&R.

\section{Summary and conclusions}

A method has been presented to simulate load induced inhomogeneous volumetric growth in a complex tissue geometry and, furthermore, to asses the effect of fixing or updating of the stress-free reference configuration. In the cardiac $\mathrm{LV}$, inhomogeneous volume changes in magnitude of $30 \%$ could be simulated by using a deviation in end-diastolic myofiber strain from its homeostatic value as stimulus. As growth progressed and the reference configurations in the two approaches started to diverge, the end-diastolic strain also became different. With an updated reference, stimulus amplitude decreased over time everywhere in the wall. However, with a fixed reference no such reduction in stimulus was observed. It is concluded that, when modeling volumetric 
growth, the choice of the reference configuration is of great importance for the computed growth.

Acknowledgments This research was financially supported by grant 2007B203 of the Netherlands Heart Foundation.

Open Access This article is distributed under the terms of the Creative Commons Attribution Noncommercial License which permits any noncommercial use, distribution, and reproduction in any medium, provided the original author(s) and source are credited.

\section{References}

Alastrue V, Pena EA, Martinez MA, Doblare M (2007) Assessing the use of the opening angle method to enforce residual stresses in patient-specific arteries. Ann Biomed Eng 35:1821-1837

Bathe KJ (1982) Finite element procedures in engineering analysis. Prentice-Hall, Englewood Cliffs

Bovendeerd PHM, Arts T, Huyge JM, van Campen DH, Reneman RS (1992) Dependence of local left ventricular wall mechanics on myocardial fiber orientation: a model study. J Biomech 25: $1129-1140$

Costa KD, Holmes JW, McCulloch AD (2001) Modeling cardiac mechanical properties in three dimensions. Phil Trans R Soc Lond A 359:1233-1250

Donker DW, Volders PGA, Arts T, Bekkers BCAM, Hofstra L, Spätjens RLHMG, Beekman JDM, Borgers M, Crijns HJGM, Vos MA (2005) End-diastolic myofiber stress and ejection strain increase with ventricular volume overload: Serial in-vivo analyses in dogs with complete atrioventricular block. Basic Res Cardiol 100:1-11

Geerts-Ossevoort L (2003) Cardiac myofiber orientation: a mechanism for adaptation? Ph.D. thesis, Eindhoven University of Technology, Eindhoven, The Netherlands

Gerdes AM, Capasso JM (1995) Structural remodeling and mechanical dysfunction of cardiac myocytes in heart failure. J Mol Cell Cardiol $27: 849-856$

Holmes JW (2004) Candidate mechanical stimuli for hypertrophy during volume overload. J Appl Physiol 97:1453-1460

Humphrey JD (2001) Stress, strain, and mechanotransduction in cells. J Biomech Eng 123:638-641

Humphrey JD, Rajagopal KR (2002) A constrained mixture model for growth and remodeling of soft tissues. Math Mod Method Appl Sci 12:407-430

Kerckhoffs RCP, Bovendeerd PHM, Kotte JCS, Prinzen FW, Smits K, Arts T (2003) Homogeneity of cardiac contraction despite physiological asynchrony of depolarization: A model study. Ann Biomed Eng 31:536-547
Kuhl E, Maas R, Himpel G, Menzel A (2007) Computational modeling of arterial wall growth: Attempts towards patient-specific simulations based on computer tomography. Biomechan Model Mechanobiol 6:321-331

MacKenna D, Summerour SR, Villarreal FJ (2000) Role of mechanical factors in modulating fibroblast function and extracellular matrix synthesis. Cardiovasc Res 46:257-263

Nash MP, Hunter PJ (2000) Computational mechanics of the heart: from tissue structure to ventricular function. J Elas 61:113-141

Omens JH (1998) Stress and strain as regulators of myocardial growth. Prog Biophys Mol Biol 69:559-572

van Oosterhout MFM, Prinzen FW, Arts T, Schreuder JJ, Vanagt WYR, Cleutjens JPM, Reneman RS (1998) Asynchronous electrical activation induces asymmetrical hypertrophy of the left ventricular wall. Circ 98:588-595

Ramasubramanian A, Latacha KS, Benjamin JM, Voronov DA, Ravi A, Taber LA (2006) Computational model for early cardiac looping. Ann Biomed Eng 34:1655-1669

Rodriguez EK, Hoger A, McCulloch AD (1994) Stress-dependent finite growth in soft elastic tissues. J Biomech 27:455-467

Rodriguez JR, Giocolea JM, Gabaldon F (2007) A volumetric model for growth of arterial walls with arbitrary geometry and loads. J Biomech 40:961-971

Sadoshima J, Izumo S (1997) The cellular and molecular response of cardiac myocytes to mechanical stress. Ann Rev Physiol 59: $551-571$

Simpson DG, Majeski M, Borg TK, Terracio L (1999) Regulation of cardiac myocyte protein turnover and myofibrillar structure in vitro by specific directions of stretch. Circ Res 85:e59-e69

Skalak E, Dasgupta G, Moss M, Otten E, Dullemeijer P, Vilmann H (1982) Analytical description of growth. J Theor Biol 94: $555-577$

Stevens C, Remme E, LeGrice IJ, Hunter P (2003) Ventricular mechanics in diastole: material parameter sensitivity. J Biomech 36:737748

Taber LA (1998) A model for aortic growth based on fluid shear and fiber stresses. J Biomech Eng 120:348-354

Taber LA, Humphrey JD (2001) Stress-modulated growth, residual stress and vascular heterogeneity. J Biomech Eng 123:528-535

Usyk TP, LeGrice IJ, McCulloch AD (2002) Computational model of three-dimensional cardiac electromechanics. Comp Visual Sci 4:249-257

Watton PN, Hill NA, Heil M (2004) A mathematical model for the growth of the abdominal aortic aneurysm. Biomechan Model Mechanobiol 3:98-113 